\title{
Ryanodine receptor 2 contributes to hemorrhagic shock-induced bi-phasic vascular reactivity in rats
}

\author{
Rong ZHOU*, Xiao-li DING, Liang-ming LIU \\ State Key Laboratory of Trauma, Burns and Combined Injury, the 2nd Department of Research Institute of Surgery, Daping Hospital, \\ the Third Military Medical University, Chongqing 400042, China
}

\begin{abstract}
Aim: Ryanodine receptor 2 (RyR2) is a critical component of intracellular $\mathrm{Ca}^{2+}$ signaling in vascular smooth muscle cells (VSMCs). The aim of this study was to investigate the role of RyR2 in abnormal vascular reactivity after hemorrhagic shock in rats.

Methods: SD rats were hemorrhaged and maintained mean arterial pressure (MAP) at $40 \mathrm{mmHg}$ for $30 \mathrm{~min}$ or $2 \mathrm{~h}$, and then superior mesenteric arteries (SMA) rings were prepared to measure the vascular reactivity. In other experiments, SMA rings of normal rats and rat VSMCs were exposed to a hypoxic medium for 10 min or $3 \mathrm{~h}$. SMA rings of normal rats and VSMCs were transfected with siRNA against RyR2. Intracellular $\mathrm{Ca}^{2+}$ release in VSMCs was assessed using Fura-2/AM.

Results: The vascular reactivity of the SMA rings from hemorrhagic rats was significantly increased in the early stage (30 min), but decreased in the late stage $(2 \mathrm{~h})$ of hemorrhagic shock. Similar results were observed in the SMA rings exposed to hypoxia for 10 min or $3 \mathrm{~h}$. The enhanced vascular reactivity of the SMA rings exposed to hypoxia for 10 min was partly attenuated by transfection with RyR2 siRNA, whereas the blunted vascular reactivity of the SMA rings exposed to hypoxia for $3 \mathrm{~h}$ was partly restored by transfection with RyR2 siRNA. Treatment with the RyR agonist caffeine ( $1 \mathrm{mmol} / \mathrm{L})$ significantly increased $\mathrm{Ca}^{2+}$ release in VSMCs exposed to hypoxia for 10 min or $3 \mathrm{~h}$, which was partially antagonized by transfection with RyR2 siRNA.

Conclusion: RyR2-mediated $\mathrm{Ca}^{2+}$ release contributes to the development of bi-phasic vascular reactivity induced by hemorrhagic shock or hypoxia.
\end{abstract}

Keywords: hemorrhagic shock; hypoxia; vascular reactivity; artery ring; vascular smooth muscle cell; RyR2; Ca ${ }^{2+}$; caffeine; siRNA

Acta Pharmacologica Sinica (2014) 35: 1375-1384; doi: 10.1038/aps.2014.83; published online 29 Sep 2014

\section{Introduction}

Vascular reactivity to vasoconstrictors, including norepinephrine (NE) and angiotensin II (Ang II), is significantly changed after severe trauma and shock (hemorrhagic or endotoxic) ${ }^{[1]}$. Our previous studies have shown that vascular reactivity to vasoconstrictors increases in the early phase and decreases significantly during late stage following hemorrhagic shock (also called bi-phasic reactivity $)^{[2]}$. However, the possible mechanisms involved in the development of vascular bi-phasic reactivity after shock remain largely unknown.

$\mathrm{Ca}^{2+}$ release from the sarcoplasmic reticulum (SR) and the sensitivity of the contractile apparatus of vascular smooth muscle cells (VSMCs) to $\mathrm{Ca}^{2+}$ are the two major mechanisms that underlie the regulation of vascular reactivity to vasoactivators and vascular tone, which is critical for maintaining peripheral blood pressure and sustaining tissue perfusion.

\footnotetext{
* To whom correspondence should be addressed.

E-mail zhourong200012@163.com

Received 2014-02-24 Accepted 2014-06-05
}

There are two types of $\mathrm{Ca}^{2+}$ release regulation receptors located in the SR: the inositol triphosphate-sensitive receptor $\left(\mathrm{IP}_{3} \mathrm{R}\right)$ and ryanodine-sensitive receptors (RyRs) ${ }^{[3]}$. Under normal conditions, NE-induced vasoconstriction associated with both the increased $\mathrm{IP}_{3} \mathrm{R}$-mediated $\mathrm{Ca}^{2+}$ release in VSMCs and the blunted RyR-mediated $\mathrm{Ca}^{2+}$ release ${ }^{[4]}$. Our previous research showed that it was RyR-mediated $\mathrm{Ca}^{2+}$ release but not $\mathrm{IP}_{3} \mathrm{R}$-mediated $\mathrm{Ca}^{2+}$ release from the SR in VSMCs that played a central role in the development of vascular dysfunction after hemorrhagic shock ${ }^{[5]}$. Three subtypes of RyR receptors (RyR1, RyR2, and RyR3) located in the SR of VSMCs have been demonstrated to be closely associated with the regulation of vascular tension ${ }^{[6,7]}$. It has been well documented that RyR2 is broadly distributed within the SR in VSMCs and that RyR2mediated $\mathrm{Ca}^{2+}$ release is over-activated in ischemic/hypoxic VSMC injury ${ }^{[8]}$. Because ischemic/hypoxic cellular injury is one of the most prominent pathophysiologic mechanisms following hemorrhagic shock, RyR2-mediated $\mathrm{Ca}^{2+}$ release in VSMCs may be over-activated in VSMCs after hemorrhagic shock. 
Although the activation of RyR2 results in $\mathrm{Ca}^{2+}$ release from the SR in VSMCs, its regulation on vascular tone is controversial. Studies have shown that the activation of RyR2-mediated $\mathrm{Ca}^{2+}$ release resulted in vasoconstriction ${ }^{[9]}$ through the $\mathrm{Ca}^{2+} /$ CaM-dependent myosin light chain (MLC) kinase (MLCK) pathway ${ }^{[10]}$. Other studies have reported that RyR2-evoked $\mathrm{Ca}^{2+}$ release could activate $\mathrm{Ca}^{2+}$-dependent potassium $\left(\mathrm{BK}_{\mathrm{Ca}}\right)$ channels, which negatively regulate vasoconstriction. In addition, RyR2-evoked $\mathrm{Ca}^{2+}$ release has been shown in our previous reports to be involved in the development of vascular hyporeactivity in the late stage after hemorrhagic shock ${ }^{[11-13]}$. Based on this evidence, we further hypothesized that RyR2 may be engaged in the occurrence of vascular bi-phasic reactivity at different stages after hemorrhagic shock.

In the current study, the superior mesenteric artery (SMA) was selected as a model for elucidating the role of RyR2 in the development of vascular bi-phasic reactivity after hemorrhagic shock. SMAs from a hemorrhagic shock rat model, hypoxiatreated SMA rings and hypoxic VSMCs were used to observe the changes of RyR2-evoked $\mathrm{Ca}^{2+}$ release from the SR and its role in the development of vascular bi-phasic reactivity at different stages after hemorrhagic shock. To our knowledge, this is the first report about the role of RyR2 in the development of vascular bi-phasic reactivity to NE after hemorrhagic shock in rats.

\section{Materials and methods}

This study was approved by the Research Council and Animal Care and Use Committee of the Research Institute of Surgery, Daping Hospital, the Third Military Medical University. All experiments conformed to the guidelines of ethical use of animals, and all efforts were made to minimize animal suffering and to reduce the number of animals used.

\section{Drugs and reagents}

The RyR agonists caffeine (Caf) and pentobarbital sodium were purchased from Sigma Co (St Louis, MO, USA). $L$-glutamine and penicillin-streptomycin were purchased from Gibco Co (BRL Co, Ltd, USA), and RyR2 siRNA, control siRNA and the siRNA transfection reagent were purchased from Santa Cruz (Dallas, TX, USA). Norepinephrine (NE) was obtained from Shanghai Harvest Pharmaceutical Co (Shanghai, China). The illustra QuickPrep Micro mRNA Purification Kit was obtained from GE Healthcare (Little Chalfont, UK), SuperScript III Reverse Transcriptase was obtained from Invitrogen/Life Technologies (Grand Island, NY, USA), and Taq DNA polymerase was obtained from Takara (Dalian, China). Fura-2/AM was obtained from Beyotime Institute of Biotechnology (Haimen, China), and Dulbecco's modified Eagle's medium (DMEM)/F12 and fetal bovine serum were obtained from HyClone Co (Logan, UT, USA).

\section{Surgical procedures and preparation of a hemorrhagic shock} model

A hemorrhagic shock rat model was established in our previous reports $^{[5]}$. Briefly, Sprague-Dawley (SD) rats (210-230 g) provided by the Animal Center of the Research Institute of Surgery, the Third Military Medical University (Chongqing, China) were anesthetized with pentobarbital sodium (40 $\mathrm{mg} / \mathrm{kg}$, iv). Then, the right femoral artery was catheterized with a polyethylene catheter for monitoring the mean arterial pressure (MAP) and bleeding. The catheter was filled with normal $(0.9 \%)$ saline containing $30 \mathrm{U} / \mathrm{mL}$ of heparin to prevent clot formation. The rats were hemorrhaged and maintained at $40 \mathrm{mmHg}$ of MAP for $30 \mathrm{~min}$ or $2 \mathrm{~h}$.

\section{Preparation of VSMCs}

VSMCs were obtained as previously described ${ }^{[2]}$. Briefly, SD rats were anesthetized using pentobarbital solution, and the SMA was removed under sterile conditions and washed several times in phosphate-buffered saline (PBS, mmol/L: $\mathrm{NaCl}$ 154, $\mathrm{Na}_{2} \mathrm{HPO}_{4} \cdot 12 \mathrm{H}_{2} \mathrm{O} 19.5, \mathrm{NaH}_{2} \mathrm{PO}_{4} \cdot 2 \mathrm{H}_{2} \mathrm{O} 3.6, \mathrm{pH}$ 7.4) to remove excessive blood cells and connective tissues. Next, the endothelium was gently scraped, and the tissues were gently cut into $0.2 \mathrm{~mm} \times 0.2 \mathrm{~mm}$ pieces. Each piece of tissue was placed in a culture bottle. Then, DMEM/F12 culture medium supplemented with inactivated $10 \%$ fetal bovine serum, streptomycin $(100 \mu \mathrm{g} / \mathrm{mL})$ and penicillin $(100 \mathrm{U} / \mathrm{mL})$ were added. Cultures were incubated in a humidified atmosphere of $5 \%$ $\mathrm{CO}_{2}$ and $95 \%$ air at $37^{\circ} \mathrm{C}$.

\section{Hypoxia treatment}

The hypoxia treatment of VSMCs or vascular rings was conducted as previously reported ${ }^{[2,14]}$. Briefly, VSMCs $\left(3 \times 10^{5}\right.$ per well) or cultured vascular rings were placed into a hypoxia culture compartment that was bubbled into an atmosphere of $95 \% \mathrm{~N}_{2}$ and $5 \% \mathrm{CO}_{2}$ at $10 \mathrm{~L} / \mathrm{min}$ for $10 \mathrm{~min}$ and then equilibrated for $10 \mathrm{~min}$. This procedure was repeated four times until the oxygen concentration in the culture compartment was less than $0.2 \%$. In the control experiments, the tissues or cells were treated as above but were exposed to a normoxic medium. The hypoxia time was based on the experimental design.

\section{RNA interference in VSMCs}

The technique for the suppression of RyR2 expression in rat VSMCs using siRNA against RyR2 has been described previously ${ }^{[15]}$. Briefly, VSMCs at $80 \%$ confluence were transfected with RyR2 siRNA using transfection reagent in DMEM/ F12 without FBS. The negative control or RyR2 siRNA was dissolved at $100 \mathrm{nmol} / \mathrm{L}$ in $100 \mathrm{~mL}$ of serum-free culture medium, mixed with $8 \mathrm{~mL}$ of transfection reagent, and incubated for $10 \mathrm{~min}$ at room temperature. The mixture was then added to the cultures with $0.9 \mathrm{~mL}$ of the culture medium, which resulted in a final siRNA concentration of $10 \mathrm{nmol} / \mathrm{L}$. Fresh growth medium was added $6 \mathrm{~h}$ after transfection, and the experiments were performed after $48 \mathrm{~h}$. Cell viability was tested by MTT assays, and the knockdown of RyR2 was confirmed by RT-PCR and immunocytochemistry.

RNA interference and reverse permeabilization in SMA rings

To downregulate the expression of RyR2 in an isolated SMA 
ring, RNA interference and reverse permeabilization was conducted to introduce control siRNA or RyR2 siRNA molecules into intact SMA rings, as previously report ${ }^{[16]}$. Briefly, RyR2 siRNA and control siRNA were dissolved at a concentration of $20 \mu \mathrm{mol} / \mathrm{L}$ in siRNA suspension buffer, following the manufacturer's instructions. To permeabilize the arteries, segments were first incubated for $20 \mathrm{~min}$ at $4{ }^{\circ} \mathrm{C}$ in the following solution (in mmol/L): $120 \mathrm{KCl}, 2 \mathrm{MgCl}_{2}, 10$ EGTA, $5 \mathrm{Na}_{2} \mathrm{ATP}$, and 20 TES ( $\mathrm{pH}$ 6.8). Arteries were then placed in a similar solution containing siRNA (final concentration: 10-50 nmol/L) for $3 \mathrm{~h}$ at $4^{\circ} \mathrm{C}$ and transferred to a third siRNA-containing solution with elevated $\mathrm{MgCl}_{2}(10 \mathrm{mmol} / \mathrm{L})$ for $30 \mathrm{~min}$ at $4{ }^{\circ} \mathrm{C}$. For reverse permeabilization, the arteries were placed in a MOPSbuffered physiological siRNA-containing solution consisting of (in mmol/L) $140 \mathrm{NaCl}, 5 \mathrm{KCl}, 10 \mathrm{MgCl}_{2}, 5$ glucose, and 2

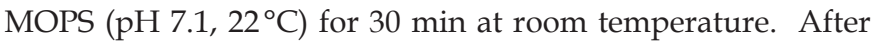
the reverse permeabilization procedures, the arteries were organ cultured for 2-3 d in DMEM/F12 culture medium supplemented with $2 \mathrm{mmol} / \mathrm{L} \mathrm{L}$-glutamine and $0.5 \%$ penicillinstreptomycin. The arteries were then used for evaluating RyR2 siRNA transfection efficiency by RT-PCR or for the detection of vascular reactivity to NE after hypoxic treatment.

\section{RyR2 RT-PCR}

$\operatorname{Poly}(\mathrm{A})^{+}$RNA was extracted from VSMCs using the illustra QuickPrep Micro mRNA Purification Kit and served as the template for cDNA synthesis with SuperScript III Reverse Transcriptase. The cDNA obtained was then amplified by RTPCR with Taq DNA polymerase. The primer pairs used were 5'-TCCAGCGATACTGCTAAAGTGACC-3' /5' -TGCATCGCTGAAATCTAGTGCAGC-3' for RyR2 and 5'-TTCTACAATGAGCTGCGTGTGG-3' / 5' -ACACAGAGTACTTGCGCTCAGGA-3' for $\beta$-actin. The PCR conditions were as follows: an initial denaturation at $95^{\circ} \mathrm{C}$ for $2 \mathrm{~min}, 40$ cycles of amplification $\left[95^{\circ} \mathrm{C}\right.$ for $30 \mathrm{~s}, 50^{\circ} \mathrm{C}$ (RyR2) or $58^{\circ} \mathrm{C}$ ( $\beta$-actin) for $30 \mathrm{~s}, 72^{\circ} \mathrm{C}$ for $50 \mathrm{~s}$, and a final extension at $72^{\circ} \mathrm{C}$ for $7 \mathrm{~min}$. The PCR products were electrophoresed in $1.5 \%$ agarose gel and stained with ethidium bromide, as previously reported ${ }^{[17]}$.

\section{Immunocytochemistry}

Cells transfected with RyR2 siRNA were washed with 0.01 mol/L PBS 3 times and fixed with $4 \%$ paraformaldehyde in PBS for $10 \mathrm{~min}$ at room temperature. Cells were then rinsed twice with PBS, incubated with PBS containing 0.5\% Triton X-100 for $5 \mathrm{~min}$, and then washed again 3 times. The cells were blocked with $0.1 \%$ BSA in PBS for $1 \mathrm{~h}$ and then incubated with primary anti-RyR2 monocolonal antibody at a dilution of 1:50 overnight at $4{ }^{\circ} \mathrm{C}$. After being washed 3 times with PBS, the cells were incubated with a FITC-tagged secondary antibody at a dilution of 1:100 in PBS at room temperature $\left(20-25^{\circ} \mathrm{C}\right)$ for $1 \mathrm{~h}$. Immunofluorescence images were obtained using a laser scanning confocal microscope (Fluoview 1000, Olympus, Japan). Excitation of FITC was accomplished by illumination at $488 \mathrm{~nm}$, and the emission was collected using a variable band-pass filter set at 500-540 nm.

\section{Measurement of $\left[\mathrm{Ca}^{2+}\right]$}

To observe the RyR-mediated $\mathrm{Ca}^{2+}$ release from the SR, cultured VSMCs from the SMA were loaded with the fluorescent $\mathrm{Ca}^{2+}$ indicator dye Fura-2/ AM $(5 \mu \mathrm{mol} / \mathrm{L})$ in normoxic PSS at room temperature $\left(20-25^{\circ} \mathrm{C}\right)$ for $30 \mathrm{~min}$, followed by washing three times with dye-free PSS. The fluorescent dye was alternatively excited at $340 \mathrm{~nm}$ and $380 \mathrm{~nm}$, and the emitted fluorescence was detected at $510 \mathrm{~nm}$ using a silicon-intensifiedtarget video camera (C2400-8, Japan) and then digitized by an image processor. The background signal was corrected by the fluorescence recorded in either non-cell regions. The Fura-2 ratio corrected for background fluorescence was converted to $\left[\mathrm{Ca}^{2+}\right]$ by the ratio between the two excitation wavelengths (340 and $380 \mathrm{~nm}$ ). Because of the recognized uncertainties inherent to the measurement of absolute $\left[\mathrm{Ca}^{2+}\right]$, the results are expressed as the R340/380 $\mathrm{nm}$ fluorescence ratio throughout this study.

\section{Measurement of vascular contraction}

Each arterial ring from the superior mesenteric rat artery was stretched to a passive force (preload) of approximately $0.6 \mathrm{~g}$ preload and equilibrated for $2 \mathrm{~h}$ in normal Krebs solution (in mmol/L: $118 \mathrm{NaCl}, 4.7 \mathrm{KCl}, 1.03 \mathrm{KH}_{2} \mathrm{PO}_{4}, 1.4 \mathrm{MgSO}_{4}$, $25 \mathrm{NaHCO}_{3}, 2.2 \mathrm{CaCl}_{2}$ and 11.5 glucose, $\mathrm{pH}$ 7.3) or Ca-free $\mathrm{K}-\mathrm{H}$ solution (substituting $\mathrm{MgCl}_{2}$ for $\mathrm{CaCl}_{2}$ in the $\mathrm{Krebs}$ solution and adding $0.2 \mathrm{mmol} / \mathrm{L}$ EGTA). Next, the solution was bubbled with $97 \% \mathrm{O}_{2}$ and $3 \% \mathrm{CO}_{2}$. The contractile response of each artery ring to NE was recorded by a Powerlab polygraph (AD instrument, Castle Hill, Australia) through a force transducer. NE was added cumulatively from $10^{-9}$ to $10^{-5} \mathrm{~mol} / \mathrm{L}$. The contractile force of each artery ring was calculated as the change of tension per $\mathrm{mg}$ tissue $(\mathrm{g} / \mathrm{mg})$. The NE cumulative dose-response curve and the maximal contraction induced by $10^{-5} \mathrm{~mol} / \mathrm{L} \mathrm{NE}\left(E_{\max }\right)$ were used to evaluate the vascular reactivity to NE.

\section{Changes of the vascular reactivity to NE from hemorrhagic shock rat and hypoxia-treated SMA \\ Vascular rings from hemorrhagic shock rat}

To exclude the neural and humoral interferences in vivo and to observe the changes in vascular reactivity to NE after hemorrhagic shock in rats, 48 rings (2-3 mm in length) from the SMAs of rats subjected to hemorrhagic shock $(40 \mathrm{mmHg}$, $30 \mathrm{~min}$ or $2 \mathrm{~h}$ ) or sham-operated control rats were randomized into 3 groups ( $n=8 /$ group): control, 30-min hemorrhagic shock, and 2-h hemorrhagic shock. The contractile response of each artery ring to $\mathrm{NE}$ was recorded in normal $\mathrm{K}-\mathrm{H}$ solution with $2.2 \mathrm{mmol} / \mathrm{L}\left[\mathrm{Ca}^{2+}\right]$ or in $\mathrm{Ca}^{2+}$-free $\mathrm{K}-\mathrm{H}$ solution.

\section{Hypoxia-treated vascular rings in vitro}

To search for a good model to mimic the hypoxic conditions of hemorrhagic shock, 48 artery rings (2-3 $\mathrm{mm}$ in length) of SMAs from rats subjected to hypoxia for $10 \mathrm{~min}$ or $3 \mathrm{~h}$ or sham-operated controls were randomized into 3 groups $(n=8 /$ group): control group, 10-min hypoxia group, and 3-h hypoxia 
group. The contractile response of each artery ring to NE was recorded in normal $\mathrm{K}-\mathrm{H}$ solution with $2.2 \mathrm{mmol} / \mathrm{L}\left[\mathrm{Ca}^{2+}\right]$ or in $\mathrm{Ca}^{2+}$-free $\mathrm{K}-\mathrm{H}$ solution.

\section{Changes of RyR2-evoked $\mathrm{Ca}^{2+}$ release in hypoxic VSMCs}

Hypoxic VSMCs or normal controls were randomly divided into 10 groups ( $n=6 /$ group): control, control+caffeine, 10-min hypoxia, 10-min hypoxia+caffeine, 10-min hypoxia+ caffeine+RyR2 siRNA, 10-min hypoxia+caffeine+control siRNA; 3-h hypoxia, 3-h hypoxia+caffeine, 3-h hypoxia+ caffeine+RyR2 siRNA, and 3-h hypoxia+caffeine+control siRNA to evaluate the changes of RyR2-mediated $\mathrm{Ca}^{2+}$ release in VSMCs subjected to hypoxia for $10 \mathrm{~min}$ or $3 \mathrm{~h}$. The RyR2 siRNA-transfected cells subjected to hypoxia treatment were incubated with caffeine $\left(10^{-3} \mathrm{~mol} / \mathrm{L}\right)$ for $5 \mathrm{~min}$ in $D$-Hank's solution. The single cell $\left[\mathrm{Ca}^{2+}\right]$ was measured using Fura-2/ AM as described above.

Involvement of RyR2 in the regulation of vascular bi-phasic reactivity to NE in hypoxia-treated SMA from rat

To explore the role of RyR2 in the regulation of vascular reactivity to NE after hemorrhagic shock, 160 artery rings (2-3 $\mathrm{mm}$ in length) of SMAs from rats subjected to hypoxia (for $10 \mathrm{~min}$ or $3 \mathrm{~h}$ ) or normal controls were randomized into 13 groups ( $n=8 /$ group): control, control+control siRNA, control+caffeine, 10-min hypoxia, 10-min hypoxia+caffeine, 10-min hypoxia+RyR2 siRNA, 10-min hypoxia+control siRNA, 10-min hypoxia+RyR2 siRNA+caffeine, 3-h hypoxia, 3-h hypoxia+caffeine, 3-h hypoxia+RyR2 siRNA, 3-h hypoxia+control siRNA, and 3-h hypoxia+RyR2 siRNA+caffeine. After transfection with RyR2 siRNA, the contractile response of each artery ring to $\mathrm{NE}$ was recorded in normal K-H solution with $2.2 \mathrm{mmol} / \mathrm{L}\left[\mathrm{Ca}^{2+}\right]$ or $\mathrm{Ca}^{2+}$-free $\mathrm{K}-\mathrm{H}$ solution after the incubation with caffeine $\left(10^{-3} \mathrm{~mol} / \mathrm{L}\right)$ for 10 $\min$.

\section{Statistical analysis}

The results are presented as the mean \pm standard error of mean (SEM). For continuous variables, Student's $t$ test was used for comparison between two groups and one-way analysis of variance (ANOVA) was used for multiple comparisons with the post-hoc Fisher's LSD test. A value of $P<0.05$ was considered significant, and $P<0.01$ was considered highly significant.

\section{Results}

Changes of the vascular reactivity to NE from hemorrhagic shock rat and hypoxia-treated SMA

First, we observed the changes of the rat SMA vascular reactivity to NE at different stages after hemorrhagic shock. Our results showed that during the early stage after hemorrhagic shock (40 $\mathrm{mmHg}$ for $30 \mathrm{~min}$ ), the SMA reactivity to NE was up-regulated significantly, characterized by an NE-induced cumulative dose-response curve that shifted upwards in either the $2.2 \mathrm{mmol} / \mathrm{L}\left[\mathrm{Ca}^{2+}\right] \mathrm{K}-\mathrm{H}$ solution or in the $\mathrm{Ca}^{2+}$ free $\mathrm{K}-\mathrm{H}$ solution. In addition, $10^{-5} \mathrm{~mol} / \mathrm{L} \mathrm{NE}$ induced the maximum contraction $\left(E_{\max }\right)$ in the $2.2 \mathrm{mmol} / \mathrm{L}\left[\mathrm{Ca}^{2+}\right] \mathrm{K}-\mathrm{H}$ solution also increased. However, at the late stage after hemorrhagic shock, the SMA vascular reactivity to NE was blunted significantly, and the NE-induced cumulative dose-response curve shifted downwards in either the $2.2 \mathrm{mmol} / \mathrm{L}\left[\mathrm{Ca}^{2+}\right] \mathrm{K}-\mathrm{H}$ solution or in the $\mathrm{Ca}^{2+}$ free $\mathrm{K}-\mathrm{H}$ solution, and the NE $\left(10^{-5} \mathrm{~mol} / \mathrm{L}\right)$-induced $E_{\max }$ decreased significantly in either the $2.2 \mathrm{mmol} / \mathrm{L}\left[\mathrm{Ca}^{2+}\right]$ $\mathrm{K}-\mathrm{H}$ solution or in the $\mathrm{Ca}^{2+}$ free $\mathrm{K}-\mathrm{H}$ solution (Figure $1 \mathrm{~A}$ and 1B).
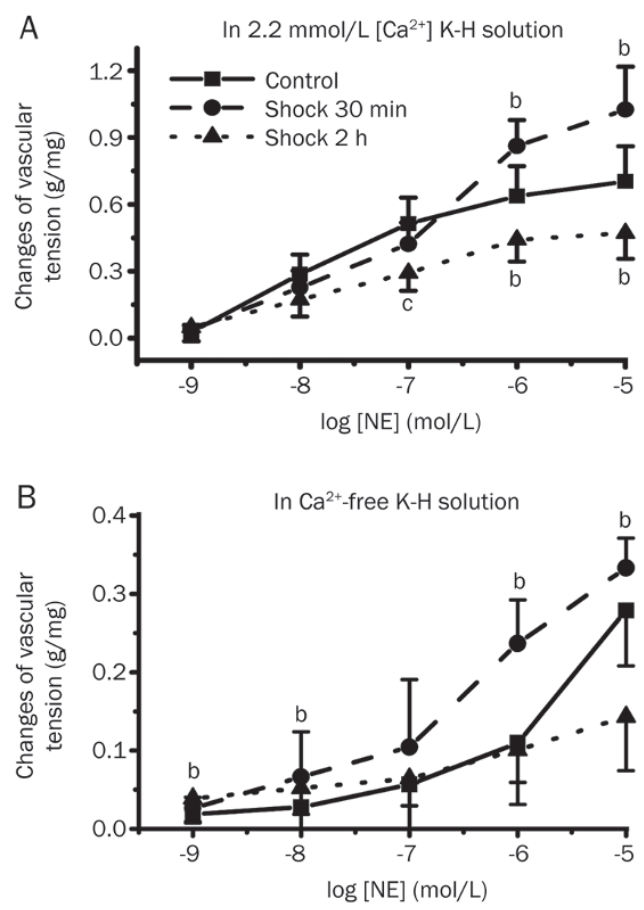

Figure 1. Changes of isolated SMA reactivity to NE after hemorrhagic shock in rats. (A) Vascular contractile reactivity to NE in normal K-H solution with $2.2 \mathrm{mmol} / \mathrm{L}\left[\mathrm{Ca}^{2+}\right]$; (B) Vascular contractile reactivity to $\mathrm{NE}$ in $\mathrm{Ca}^{2+}$-free $\mathrm{K}-\mathrm{H}$ solution. Values are the mean $\pm \mathrm{SEM}$, and there are 8 observations in each group. ${ }^{b} P<0.05,{ }^{c} P<0.01$ vs control group. NE, norepinephrine.

Next, we explored whether different extents of hypoxia in SMA rings could mimic the bi-phasic reactivity of SMA to $\mathrm{NE}$ at different stages after hemorrhagic shock in vitro. Our results showed that in hypoxic SMA rings, the vascular reactivity to NE increased significantly following hypoxia for 10 min compared with controls, and the NE-induced cumulative dose-response curve shifted upwards in either the $2.2 \mathrm{mmol} / \mathrm{L}$ $\left[\mathrm{Ca}^{2+}\right] \mathrm{K}-\mathrm{H}$ solution or in the $\mathrm{Ca}^{2+}$ free $\mathrm{K}-\mathrm{H}$ solution. The NE $\left(10^{-5} \mathrm{~mol} / \mathrm{L}\right)$-induced $E_{\max }$ significantly increased in the 2.2 $\mathrm{mmol} / \mathrm{L}\left[\mathrm{Ca}^{2+}\right] \mathrm{K}-\mathrm{H}$ solution. By contrast, vascular reactivity to NE decreased significantly after the arteries were exposed to hypoxia for $3 \mathrm{~h}$, characterized by a downward shift of the NE-cumulative dose-response curve and a significant decrease in the $E_{\max }\left(10^{-5} \mathrm{~mol} / \mathrm{L} \mathrm{NE}\right)$ in both the $2.2 \mathrm{mmol} / \mathrm{L}\left[\mathrm{Ca}^{2+}\right] \mathrm{K}-\mathrm{H}$ solution and the $\mathrm{Ca}^{2+}$ free $\mathrm{K}-\mathrm{H}$ solution (Figure 2A and $2 \mathrm{~B}$ ). 
A
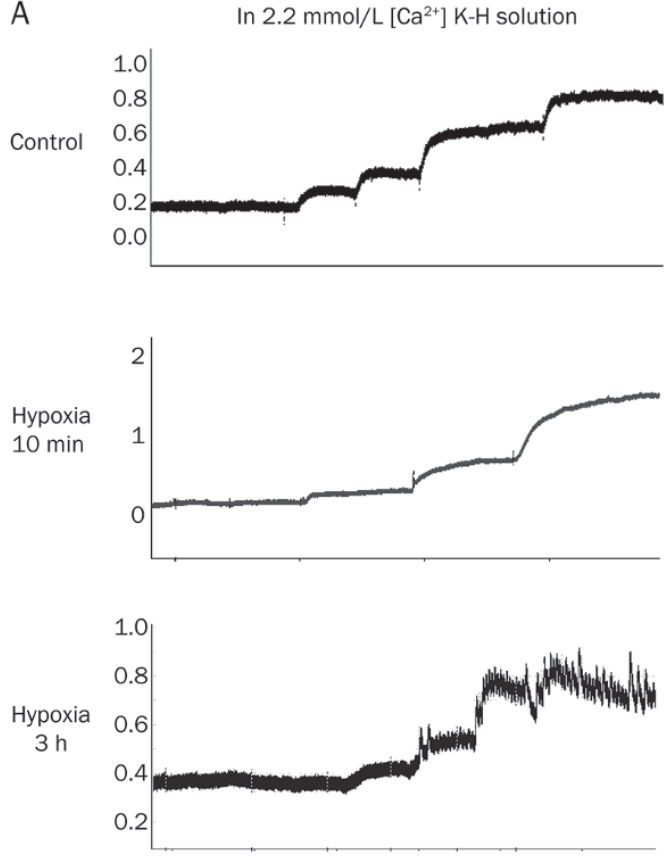

B

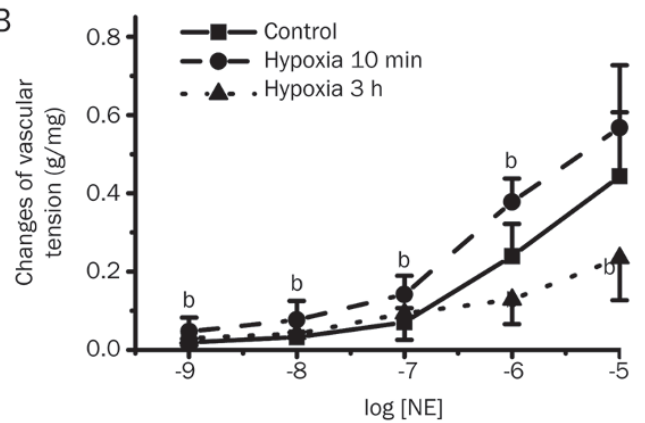

In $\mathrm{Ca}^{2+}$-free $\mathrm{K}-\mathrm{H}$ solution
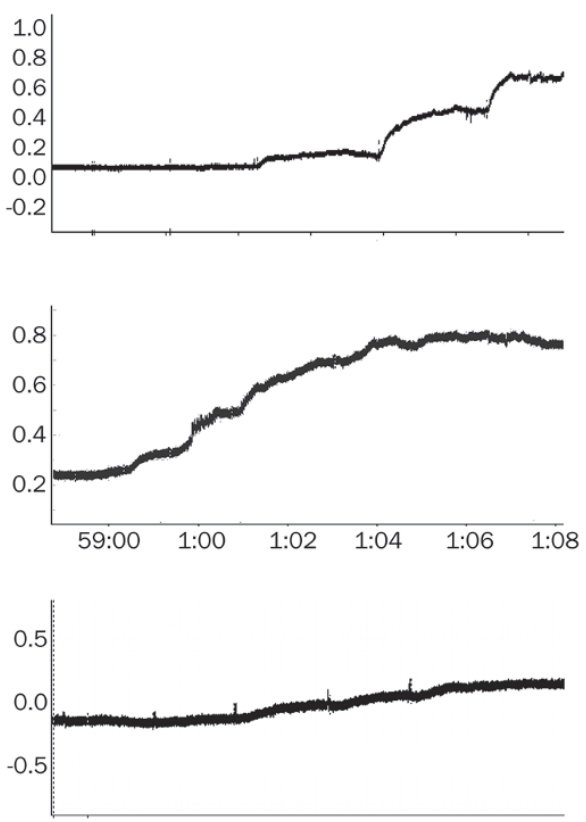

C

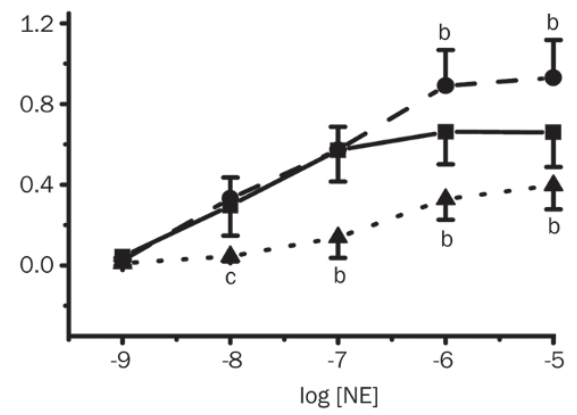

Figure 2. Changes of vascular reactivity to NE in hypoxic isolated SMAs from rats. (A) The original force recording traces of normal and hypoxic SMA

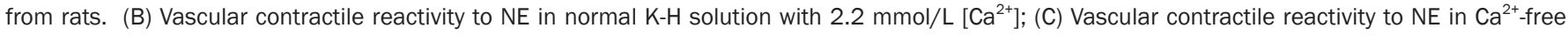
$\mathrm{K}-\mathrm{H}$ solution. Values are the mean $\pm \mathrm{SEM}$, and there are 8 observations in each group. ${ }^{\mathrm{b}} P<0.05,{ }^{\mathrm{c}} P<0.01$ vs control group. NE, norepinephrine.

\section{Changes of RyR2-mediated $\mathrm{Ca}^{2+}$ release in hypoxia-treated VSMCs}

To explore the changes of RyR2-mediated $\mathrm{Ca}^{2+}$ release from the SR in VSMCs after hemorrhagic shock, we further explored the changes of caffeine-induced, RyR2-mediated $\mathrm{Ca}^{2+}$ release in hypoxic VSMCs transfected with RyR2 siRNA. The results showed that transfection of RyR2 siRNA (10 nmol/L) could significantly inhibit the expression of RyR2 in VSMCs (Figure $3 \mathrm{~A}-3 \mathrm{C})$. In addition, compared with normal controls, the $\left[\mathrm{Ca}^{2+}\right]$ increased significantly in VSMCs subjected to hypoxia for $3 \mathrm{~h}$. Caffeine $\left(10^{-3} \mathrm{~mol} / \mathrm{L}\right)$ significantly increased the $\left[\mathrm{Ca}^{2+}\right]$ in VSMCs subjected to hypoxia for $10 \mathrm{~min}$ and $3 \mathrm{~h}$. Transfection with RyR2 siRNA could significantly attenuate caffeineinduced $\mathrm{Ca}^{2+}$ release in VSMCs subjected to hypoxia for 10 min or $3 \mathrm{~h}$ (Figure 3D-3F), whereas transfection with control siRNA had no significant influence on caffeine-triggered, RyRmediated $\mathrm{Ca}^{2+}$ release.
Involvement of RyR2 in the regulation of vascular bi-phasic reactivity to NE in SMA subjected to hypoxia

To explore the role of RyR2 in the development of vascular bi-phasic reactivity after hemorrhagic shock, the efficiency of RyR2 siRNA transfection for knocking down the expression of RyR2 in the vascular rings was evaluated by RT-PCR. The results showed that transfection of RyR2 siRNA (10, 50 nmol/L) could inhibit the expression of RyR2 (Figure 4A). The vascular reactivity to NE of SMAs increased when subjected to $10 \mathrm{~min}$ of hypoxia but decreased after $3 \mathrm{~h}$ of hypoxia. Transfection of RyR2 siRNA (10 nmol/L) significantly antagonized the enhanced vascular reactivity to NE in SMAs subjected to $10 \mathrm{~min}$ of hypoxia, as evidenced by the NE cumulative dose-response curve shifting downwards and the $10^{-5}$ mol/L NE induced the maximum contraction $\left(E_{\max }\right)$ decreasing significantly $(P<0.05$, Figure $4 \mathrm{~B})$. Moreover, preincubation with the nonselective RyR agonist caffeine $\left(10^{-3} \mathrm{~mol} / \mathrm{L}\right.$ for 10 
A

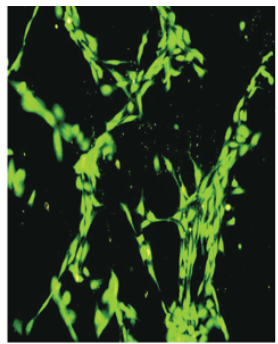

Control siRNA

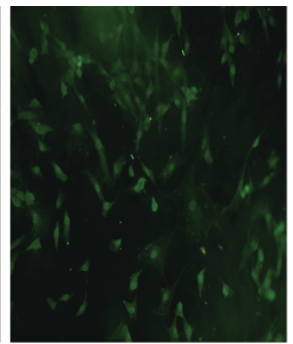

RyR2 siRNA
$B$

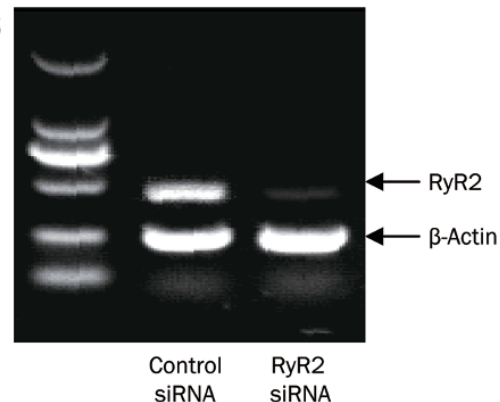

C

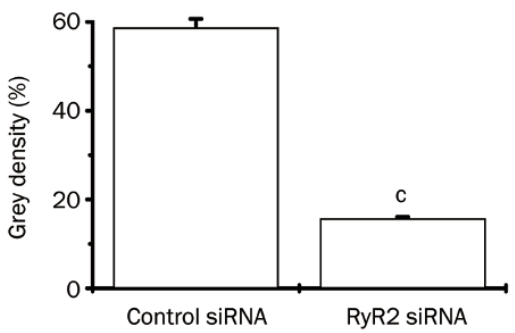

D

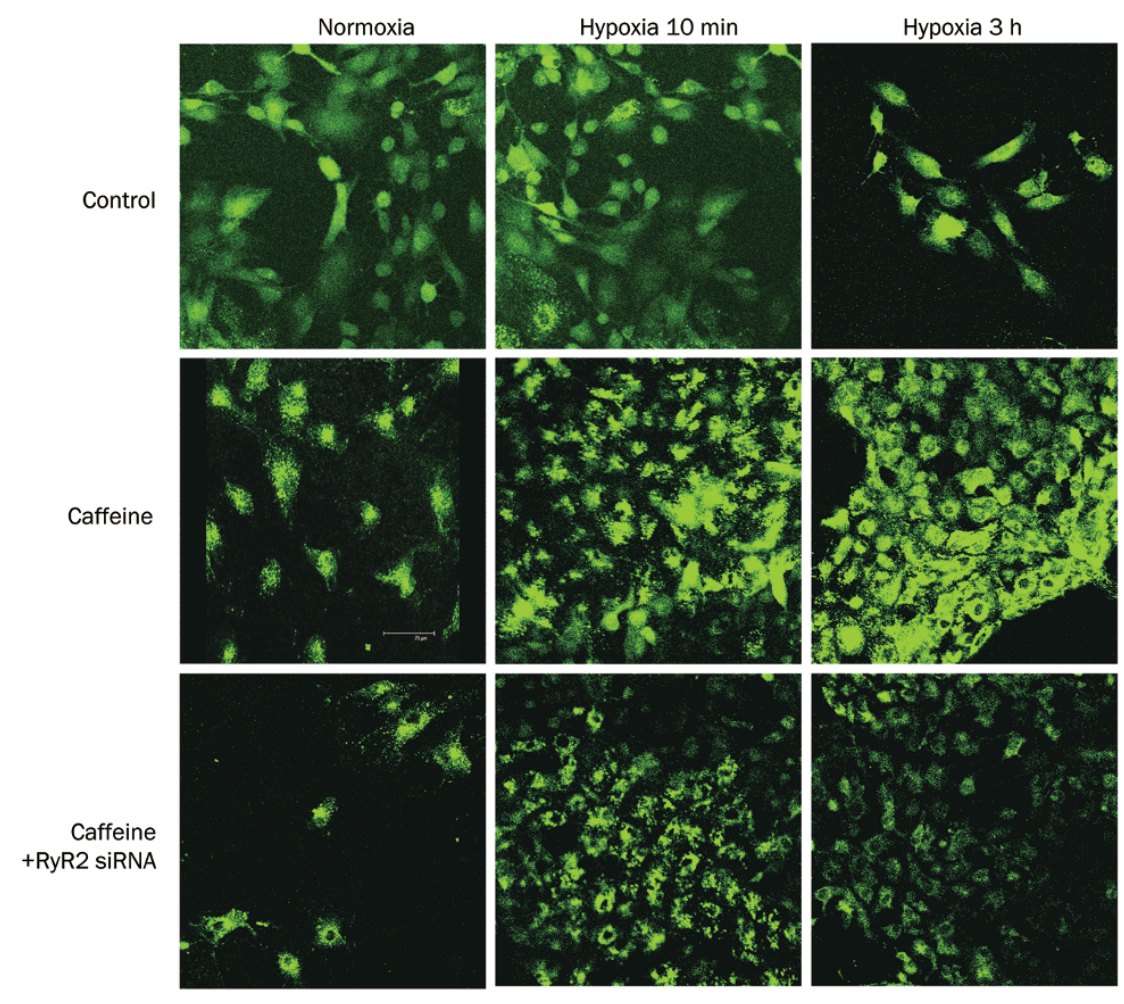

E

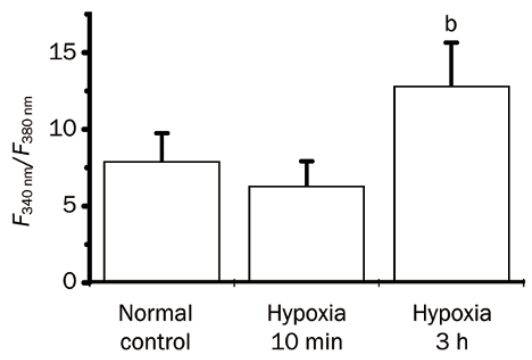

F

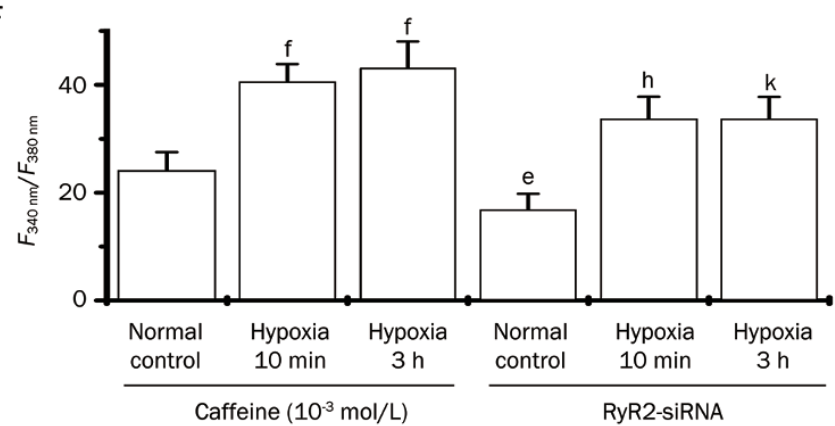

Figure 3. Effects of RyR2 siRNA transfected into VSMCs on caffeine-induced $\mathrm{Ca}^{2+}$ release from the SR. (A) Knockdown efficiency of RyR2 siRNA in VSMC cultures. The observation of RyR2 expression in cultured VSMCs transfected with RyR2 siRNA through a fluorescence microscope ( $\times 200)$. Cells were incubated with RyR2 monoclonal antibody and FITC-labeled secondary antibody; cellular fluorescence was captured using a fluorescence microscope; (B) Knockdown efficiency of RyR2 siRNA in VSMCs. After negative control siRNA or RyR2 siRNA was transfected into VSMCs using an siRNA transfection agent, RyR2 expression levels were analyzed using RT-PCR. (C) The values were normalized to those obtained under control conditions. (D) Images of intracellular free $\mathrm{Ca}^{2+}$ loaded with the fluorescent $\mathrm{Ca}^{2+}$ indicator dye Fura-2/AM in VSMCs ( $\left.\times 400\right)$. (E) Changes of [Ca ${ }^{2+}$ in hypoxic VSMCs. (F) Involvement of RyR2-mediated $\mathrm{Ca}^{2+}$ release from the SR in hypoxic VSMCs. The values were normalized to those obtained under control conditions. Values are the mean \pm SEM, and there are 5 observations in each group. ${ }^{b} P<0.05,{ }^{c} P<0.01$ vs control group. ${ }^{e} P<0.05,{ }^{f} P<0.01$ vs control+caffeine $\left(10^{-3}\right.$ mol/L) group. ${ }^{\mathrm{h}} \mathrm{P}<0.05$ vs 10 min hypoxia+caffeine group. ${ }^{k} P<0.05$ vs $3 \mathrm{~h}$ hypoxia+caffeine group. 
A

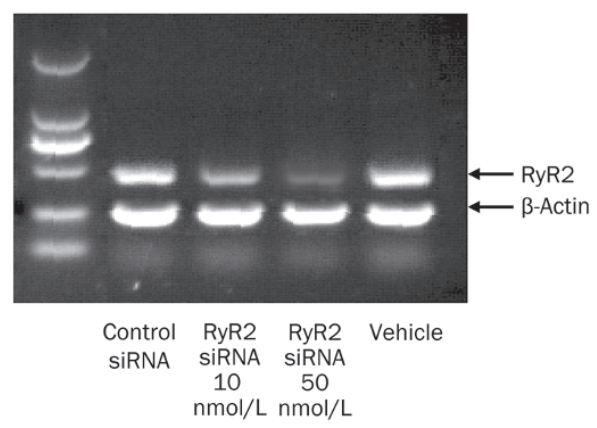

B a
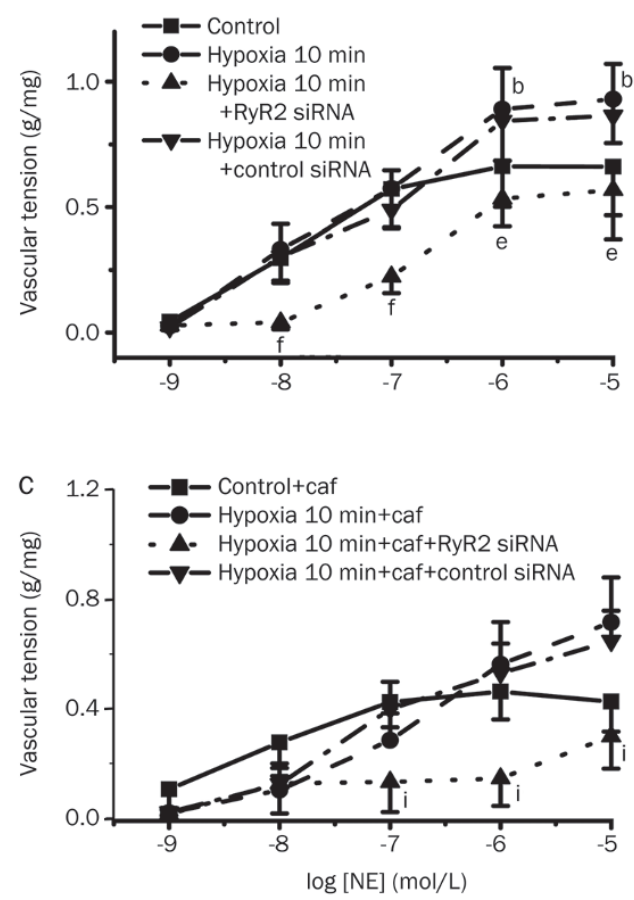

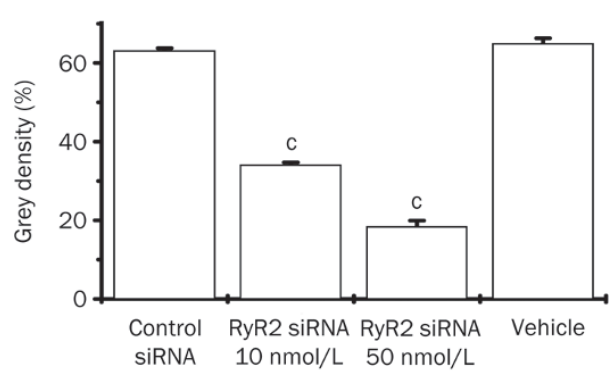

b
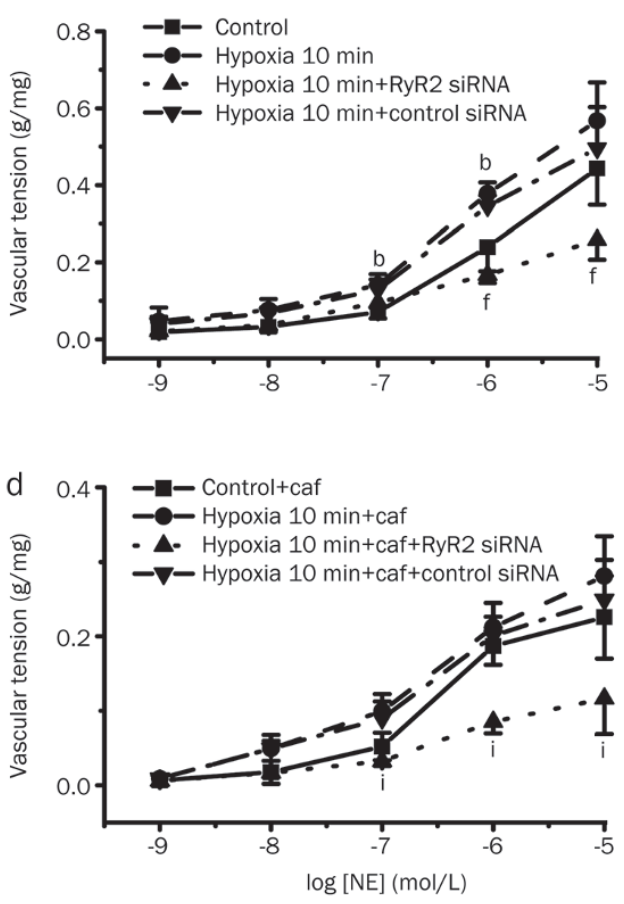

Figure 4. Involvement of RyR2 in vascular hyper-reactivity during the early stage after hemorrhagic shock. (A) Knockdown efficiency of RyR2 siRNA in superior mesenteric artery rings. After control siRNA or RyR2 siRNA was transfected into the vascular rings with a reverse permeabilization transfection method, RyR2 mRNA levels were analyzed using RT-PCR. The values were normalized by those obtained under control conditions. Values were the mean \pm SEM, and there are 4 observations in each group. ${ }^{\circ} P<0.01$ vs control group. (B) Influence of siRyR2 transfection on vascular hyper-reactivity during the early stage after hemorrhagic shock. (a) Effects of RyR2 siRNA transfection on vascular reactivity after hypoxia for 10 min in normal K-H solution; (b) Effects of RyR2 siRNA transfection on vascular reactivity after hypoxia for 10 min in $\mathrm{Ca}^{2+}$-free $\mathrm{K}$-H solution; (c) Effects of RyR2 siRNA transfection and caffeine on vascular reactivity after hypoxia for $10 \mathrm{~min}$ in normal K-H solution; (d) Effects of RyR2 siRNA transfection and caffeine on vascular reactivity after hypoxia for $10 \mathrm{~min}$ in $\mathrm{Ca}^{2+}$-free $\mathrm{K}-\mathrm{H}$ solution. Values are the mean $\pm \mathrm{SEM}$, and there are 8 observations in each group. ${ }^{\mathrm{b}} \mathrm{P}<0.05$, ${ }^{c} P<0.01$ vs control group. ${ }^{e} P<0.05,{ }^{f} P<0.01$ vs 10 min hypoxia group. ${ }^{i} P<0.01$ vs 10 min hypoxia+caffeine group.

min) resulted in no significant upregulation in the vascular reactivity of SMAs to NE. Transfection with RyR2 siRNA resulted in decreased vascular reactivity to NE in SMAs subjected to $10 \mathrm{~min}$ of hypoxia, as indicated by the NE cumulative dose-response curve shifting downwards and the $E_{\max }$ decreasing significantly $(P<0.01$, Figure $4 \mathrm{Bc}$ and $4 \mathrm{Bd})$. However, the vascular reactivity of the SMA rings to NE decreased significantly after 3-h hypoxia treatment, and transfection with RyR2 siRNA $(10 \mathrm{nmol} / \mathrm{L})$ partially but significantly restored the decreased vascular reactivity to NE, as characterized by the NE cumulative dose-response curve shifting upwards and the significant increase in $E_{\max }(P<0.01$, Figure 5A and 5B). Pre- incubation with caffeine $\left(10^{-3} \mathrm{~mol} / \mathrm{L}\right)$ decreased the vascular reactivity of hypoxia-treated SMAs to NE, which was further exacerbated by transfection with RyR2 siRNA (Figure 5C and $5 \mathrm{D})$.

\section{Discussion}

Our results showed that the vascular reactivity to NE is significantly increased during the early stage of hemorrhagic shock and significantly decreased after prolonged hemorrhagic shock, which is consistent with our previous report ${ }^{[2]}$. As hypoxia is one of the major factors contributing to the pathogenesis of hemorrhagic shock, to establish a valid model 
A
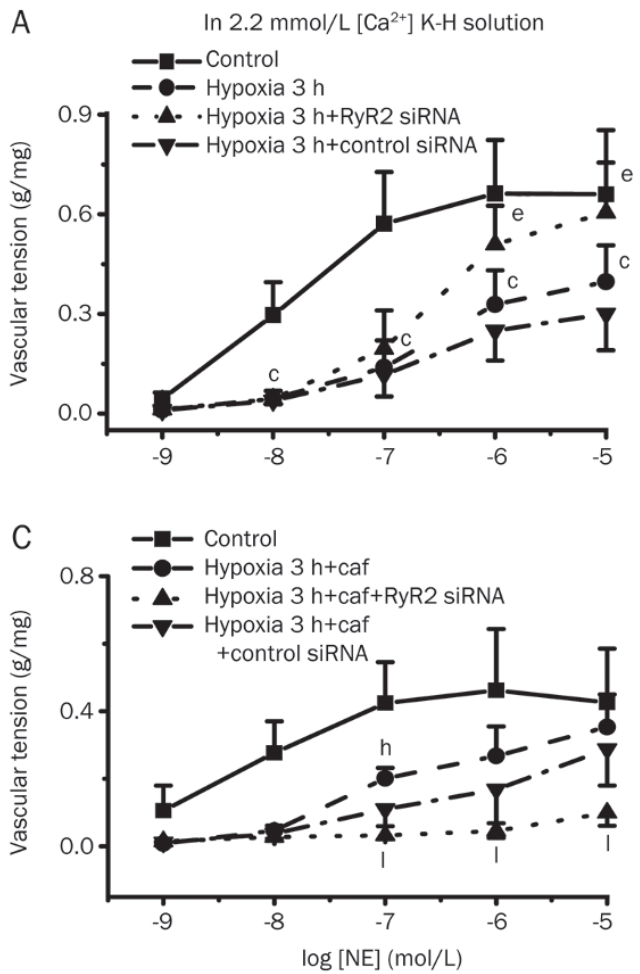

B
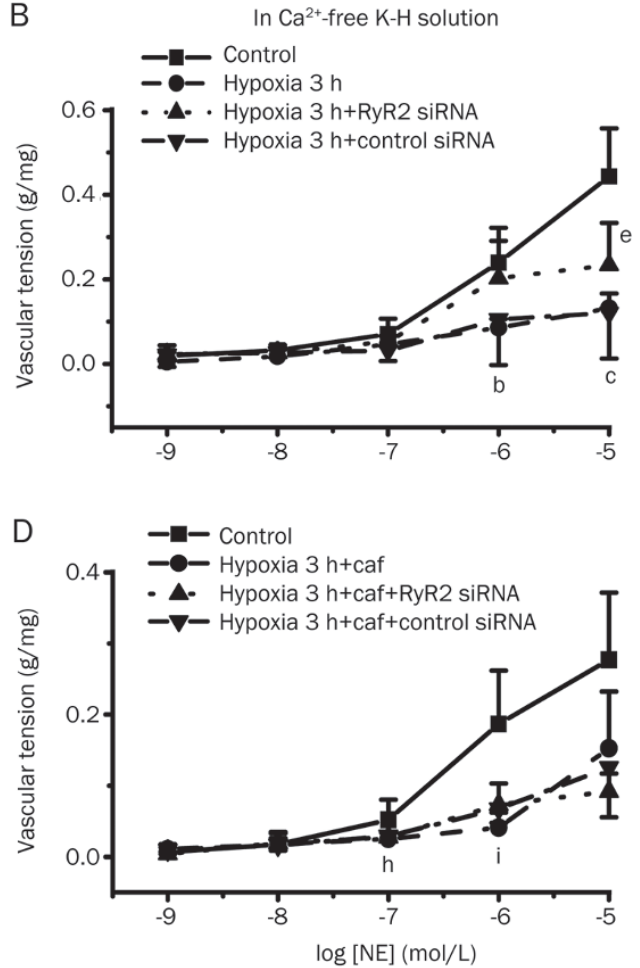

Figure 5. Involvement of RyR2 in vascular hypo-reactivity during the late stage after hemorrhagic shock. (A) Effects of RyR2 siRNA transfection on vascular reactivity after hypoxia treatment for $3 \mathrm{~h}$ in normal K-H solution; (B) Effects of RyR2 siRNA transfection on vascular reactivity after hypoxia treatment for $3 \mathrm{~h}$ in $\mathrm{Ca}^{2+}$-free $\mathrm{K}-\mathrm{H}$ solution; (C) Effects of RyR2 siRNA transfection and caffeine on vascular reactivity after hypoxia treatment for $3 \mathrm{~h}$ in normal K-H solution; (D) Effects of RyR2 siRNA transfection and caffeine on vascular reactivity after hypoxia treatment for $3 \mathrm{~h}$ in $\mathrm{Ca}^{2+}{ }^{2+}$ free $\mathrm{K}^{-\mathrm{H}}$ solution. Values are the mean \pm SEM, and there are 8 observations in each group. ${ }^{b} P<0.05,{ }^{c} P<0.01$ vs control group. ${ }^{\text {e }} P<0.05$ vs $3 \mathrm{~h}$ hypoxia group. ${ }^{\text {h }} P<0.05$, ${ }^{i} P<0.01$ vs control+caffeine group. ' $P<0.01$ vs 3 h hypoxia + caffeine group.

imitating the changes of vascular reactivity after hemorrhagic shock in vitro, the changes of hypoxic SMA artery reactivity were observed first in the current study. Our results showed that this so-called "vascular bi-phasic reactivity" after hemorrhagic shock could at least be partly imitated in hypoxic vascular rings.

RyR has been shown to be involved in NE-induced vasoconstriction. One report showed that NE induces vasoconstriction associated with RyR-mediated $\mathrm{Ca}^{2+}$ release under normal conditions. The RyR antagonist ruthenium red was shown to attenuate approximately $50 \%$ of NE-triggered vasocontraction in rat renal artery ${ }^{[18]}$. Another report showed that NEinduced vasoconstriction was associated with blunted RyRmediated $\mathrm{Ca}^{2+}$ release $^{[4]}$. Defects in RyR2, which is localized to the SR in VSMCs, are involved in many diseases and contribute to muscular dystrophy and heart failure ${ }^{[19-21]}$. It has been reported that RyR2-mediated $\mathrm{Ca}^{2+}$ release is over-activated in ischemic/hypoxic VSMC injury, which is one of the most important mechanisms involved in vascular contraction and vasoreactivity regulation after hemorrhagic shock. Whether RyR2-mediated $\mathrm{Ca}^{2+}$ release is associated with the development of vascular bi-phasic reactivity after hemorrhagic shock remained a question in the field.

In the current study, caffeine $\left(10^{-3} \mathrm{~mol} / \mathrm{L}\right)$ was used to acti- vate RyR2-mediated $\mathrm{Ca}^{2+}$ release from the SR. As a classic RyR agonist, caffeine can activate all RyR isoforms without selectivity at concentrations above $5 \times 10^{-3} \mathrm{~mol} / \mathrm{L}^{[22-24]}$, but $10^{-3}$ $\mathrm{mol} / \mathrm{L}$ of caffeine activates RyR2 $>\mathrm{RyR} 1^{[24]}$ and RyR3 $>\mathrm{RyR} 1^{[25]}$, and can increase the frequency of $\mathrm{Ca}^{2+}$ spark ${ }^{[26]}$. In addition, $\mathrm{Ca}^{2+}$ release induced by caffeine is positively related to the expression of RyR2, whereas it is negatively related to the expression of RyR3 ${ }^{[27]}$. Our results showed that caffeine $\left(10^{-3}\right.$ $\mathrm{mol} / \mathrm{L}$ )-triggered $\mathrm{Ca}^{2+}$ release from the SR was augmented in VSMCs treated with hypoxia for $10 \mathrm{~min}$ or $3 \mathrm{~h}$, whereas transfection with RyR2 siRNA could partially but significantly antagonize this effect in both groups, which suggested that RyR2-mediated $\mathrm{Ca}^{2+}$ release may be over-activated after hemorrhagic shock in either the early stage $(30 \mathrm{~min})$ or the late stage $(2 \mathrm{~h})$.

It is very interesting that although the RyR2-mediated $\mathrm{Ca}^{2+}$ release from the SR was over-activated in VSMCs treated with hypoxia for $10 \mathrm{~min}$ or $3 \mathrm{~h}$, the vascular reactivity to $\mathrm{NE}$ is notably different during the early and late stages after hemorrhagic shock. Some reports have shown that local RyR2-mediated $\mathrm{Ca}^{2+}$ release plays an important role in the modulation of vasoconstriction, whereas others reported that local RyR2mediated $\mathrm{Ca}^{2+}$ release (known as $\mathrm{Ca}^{2+}$ spark in VSMCs) negatively regulated vascular tone through the activation of the 
$\mathrm{BK}_{\mathrm{Ca}}$ channel ${ }^{[10,12]}$. Therefore, we further evaluated whether the over-activation of RyR2-mediated $\mathrm{Ca}^{2+}$ release from the SR at different stages after hemorrhagic shock was involved in vascular bi-phasic reactivity to NE.

Our results showed that in the early stage after hemorrhagic shock, although activating RyR with caffeine $\left(10^{-3} \mathrm{~mol} / \mathrm{L}\right)$ could not augment the increased vascular reactivity to $\mathrm{NE}$, the inhibition RyR2-mediated $\mathrm{Ca}^{2+}$ release with RyR2 siRNA could significantly restore the vascular hyperreactivity to NE in an SMA ring treated with hypoxia for $10 \mathrm{~min}$. However, activating RyR2 with caffeine $\left(10^{-3} \mathrm{~mol} / \mathrm{L}\right)$ further exacerbated the decreased vasoreactivity to NE in SMA rings subjected to hypoxia for $3 \mathrm{~h}$, whereas inhibition of RyR2-mediated $\mathrm{Ca}^{2+}$ release from the SR by transfection with RyR2 siRNA significantly restored the vasoreactivity to NE. Taken together, these results suggested that the over-activation of RyR2 is closely associated with the development of vascular bi-phasic reactivity to NE after hemorrhagic shock.

It is widely accepted that the primary regulatory pathway for vascular smooth muscle contraction is through the $\mathrm{Ca}^{2+}$ and calmodulin-dependent reversible phosphorylation of the $20000-D a$ myosin light chain (MLC20) ${ }^{[28]}$. In VSMCs, freeCaM binding with $\mathrm{Ca}^{2+}$ could accelerate the formation of the CaM-CaM related kinase II (CaMK II) complex, a ubiquitous multifunctional serine/threonine kinase expressed in VSMCs as multimers of $\gamma$ - and/or $\delta$-sun units ${ }^{[29]}$, and increase MLCK activity and MLC20 phosphorylation, which contribute to vascular contraction ${ }^{[30]}$. However, $\mathrm{Ca}^{2+}$ release located next to cytomembranes, also known as $\mathrm{Ca}^{2+}$ spark, triggers the formation of STOCs ${ }^{[31]}$ and activates the large conductance calcium activated potassium channel $\left(\mathrm{BK}_{\mathrm{Ca}}\right)$, which at least partially contributes to the vascular hyporeactivity observed after hemorrhagic shock ${ }^{[32]}$. However, more research is required to determine whether the over-activation of RyR2-mediated $\mathrm{Ca}^{2+}$ release during the early stage after hemorrhagic shock is coupled with the activation of CaM-CaMK II signal cascade and vascular hyperreactivity or whether the over-activation of RyR2-mediated $\mathrm{Ca}^{2+}$ release during the late stage after hemorrhagic shock is linked to the $\mathrm{BK}_{\mathrm{Ca}}$-dependent signaling pathway and the occurrence of vascular hyporeactivity.

In recent years, $\mathrm{Ca}^{2+}$ release from the SR was shown to trigger extracellular $\mathrm{Ca}^{2+}$ influx, which was also named storeoperated $\mathrm{Ca}^{2+}$ entry $(\mathrm{SOCE})^{[13]}$. In the present study, the role of RyR2-mediated $\mathrm{Ca}^{2+}$ release in the modulation of vascular reactivity to $\mathrm{NE}$ after hemorrhagic shock was observed not only in normal $\mathrm{K}-\mathrm{H}$ solution but also in $\mathrm{Ca}^{2+}$-free $\mathrm{K}-\mathrm{H}$ solution, which excluded the influence of SOCE on vascular reactivity. In this study, to exclude the neural and humoral interference in vivo, the hypoxia-induced bi-phasic change in SMA rings was examined. Our results showed that hypoxia-treated SMA rings in vitro could at least partially imitate the hypoxicischemic condition of shock. Nevertheless, owing to the limitation that this hypoxia model could only partially mimic the shocked state, a more appropriate model is needed to mimic the conditions of shock in future research. Furthermore, the hypoxic and NE responses are complex, involving many dif- ferent pathways of $\mathrm{Ca}^{2+}$ release, entry and removal. Thus, other cellular and molecular mechanisms responsible for their roles in the development of vascular bi-phasic reactivity after hemorrhagic shock could not be totally excluded.

\section{Acknowledgements}

This project was supported by National Natural Science Foundation of China (No 81100227 and 81370427) and the Key Project of Natural Science Foundation of Chongqing (No 2010BC5126).

\section{Author contribution}

Rong ZHOU designed the research, analyzed data, wrote the paper and carried out the experiments; Xiao-li DING created the model and carried out measurements of vascular reactivity; Liang-ming LIU conceived the study and participated in its design and coordination. All authors approved the final manuscript.

\section{References}

1 Liu LM, Wand JA, Dubick MA. Hemorrhage-induced vascular hyporeactivity to norepinephrine in select vasculatures of rats and the role of nitric oxide and endothelin. Shock 2003; 19: 208-14.

2 Li T, Liu L, Xu J, Yang G, Ming J. Changes of Rho kinase activity after hemorrhagic shcok and its role in shock-induced biphasic response of vascular reactivity and calcium sensitivity. Shock 2006; 26: 504-9.

3 Curtis TM, Scholfield C, McGeown DJ. Calcium signaling in ocular arterioles. Crit Rev Eukaryot Gene 2007; 17: 1-12.

4 Eckert RE, Karsten AJ, Vtz J, Ziegler M. Regulation of renal artery smooth muscle tone by $\alpha 1$-adrenoceptors: role of voltage-gated calcium channels and intracellular calcium stores. Urol Res 2000; 28: $122-7$

5 Zhou R, Chen F, Li Q, Hu DY, Liu LM. Stimulation of the adenosine A3 receptor reverses vascular hyporeactivity after hemorrhagic shock in rats. Acta Pharmacol Sin 2010; 31: 413-20.

6 Arendshorst WJ, Thai TL. Regulation of the renal microcirculation by ryanodine receptors and calcium-induced calcium release. Curr Opin Nephrol Hypertens 2009; 18: 40-9.

7 Clark JH, Kinnear NP, Kalujnaia S, Cramb G, Fleischer S, Jeyakumar $\mathrm{LH}$, et al. Identification of functionally segregated sarcoplasmic reticulum calcium stores in pulmonary arterial smooth muscle. J Biol Chem 2010; 285: 13542-9.

8 Liao B, Zheng YM, Yadav VR, Korde AS, Wang YX. Hypoxia induces intracellular $\mathrm{Ca}^{2+}$ release by causing reactive oxygen species-mediated dissociation of FK506-binding protein 12.6 from ryanodine receptor 2 in pulmonary artery myocytes. Antioxid Redox Signal 2011; 14 : 37-47.

9 McCarron JG, Chalmers S, Bradley KN, MacMillan D, Muir TC. $\mathrm{Ca}^{2+}$ microdomains in smooth muscle. Cell Calcium 2006; 40: 461-93.

10 Himpens B, Missiaen L, Casteels R. $\mathrm{Ca}^{2+}$ homeostasis in vascular smooth muscle. J Vasc Res 1995; 32: 207-19.

11 Burdyga T, Wray S. Activation potential refractory period in ureter smooth muscle is set by Ca sparks and BK channels. Nature 2005; 436: 559-62.

$12 \mathrm{Ji} \mathrm{G}$, Feldman ME, Greene KS, Sorrentino V, Xin HB, Kotlikoff MI. RyR2 proteins contribute to the formation of $\mathrm{Ca}^{2+}$ sparks in smooth muscle. J Gen Physiol 2004; 123: 377-86.

13 Munoz E, Valero RA, Quintana A, Hoth M, Nunez L, Villalobos C. Nonsteroidal anti-inflammatory drugs inhibit vascular smooth muscle 
cell proliferation by enabling the $\mathrm{Ca}^{2+}$-dependent inactivation of calcium release-activated calcium/orai channels normally prevented by mitochondria. J Biol Chem 2011; 286: 16186-96.

14 Xu J, Lan D, Li T, Yang G, Liu L. Angiopoietins regulate vascular reactivity after hemorrhagic shock in rats through the Tie2-nitric oxide pathway. Cardiovasc Res 2012; 96: 308-19.

15 Min CK, Yeom DR, Lee KE, Kwon HK, Kang M, Kim YS, et al. Coupling of ryanodine receptor 2 and voltage-dependent anion channel 2 is essential for $\mathrm{Ca}^{2+}$ transfer from the sarcoplasmic reticulum to the mitochondria in the heart. Biochem J 2012; 447: 371-9.

16 Gonzales AL, Amberg GC, Earley S. $\mathrm{Ca}^{2+}$ release from the sarcoplasmic reticulum is required for sustained TRPM4 activity in cerebral artery smooth muscle cells. Am J Physiol Cell Physiol 2010; 299: C279-88.

17 Ohashi R, Sakata SI, Naito A, Hirashima N, Tanaka M. Dendritic differentiation of cerebellar Purkinje cells is promoted by ryanodine receptors expressed by Purkinje and granule cells. Dev Neurobiol 2013; 4: 1-14.

18 Thai TL, Fellner SK, Arendshorst WJ. ADP-ribosyl cyclase and ryanodine receptor activity contribute to basal renal vasomotor tone and agonist-induced renal vasoconstriction in vivo. Am J Physiol Renal Physiol 2007; 293: F1107-14.

19 Morel JL, Dabertrand F, Fritz N, Henaff M, Mironneau J, Macrez N. The decrease of expression of ryanodine receptor sub-type 2 is reversed by gentamycin sulphate in vascular myocytes from $\mathrm{mdx}$ mice. J Cell Mol Med 2009; 13: 3122-30.

20 Shannon TR. Ryanodine receptor $\mathrm{Ca}^{2+}$ sensitivity and excitationcontraction coupling in muscular dystrophy and heart failure: similar and yet different. Am J Physiol Heart Circ Physiol 2009; 297: H19656.

21 Earley S, Heppner TJ, Nelson MT, Brayden JE. TRPV4 forms a novel $\mathrm{Ca}^{2+}$ signaling complex with ryanodine receptors and $\mathrm{BK}_{\mathrm{ca}}$ channels. Circ Res 2005; 97: 1270-9.

22 Nakai J, Ogura T, Protasi F, Franzini-Armstrong C, Allen PD, Beam
KG. Functional nonequality of the cardiac and skeletal ryanodine receptors. Proc Natl Acad Sci U S A 1997; 94: 1019-22.

23 Perez CF, Voss A, Pessah IN, Allen PD. RyR1/RyR3 chimeras reveal that multiple domains of RyR1 are involved in skeletal-type E-C coupling. Biophysical J 2003; 84: 2655-63.

24 Copello JA, Barg S, Sonnleitner A, Porta M, Diaz-Sylvester P, Fill M, et al. Different activation by $\mathrm{Ca}^{2+}$, ATP and caffeine of cardiac and skeletal muscle ryanodine receptors after block by $\mathrm{Mg}^{2+}$. J Member Biol 2002; 187: 51-64.

25 Fessenden JD, Wang Y, Moore RA, Chen SR, Allen PD, Pessah IN. Divergent functional properties of ryanodine receptor type 1 and 3 expressed in a myogenic cell line. Biophys J 2000; 79: 2509-25.

26 Trafford AW, Sibbring GC, Diaz ME, Eisner DA. The effects of low concentrations of caffeine on spontaneous Ca release in isolated rat ventricular myocytes. Cell Calcium 2000; 28: 269-76.

27 Zheng YM, Wang QS, Liu QH, Rathore R, Yadav V, Wang YX. Heterogeneous gene expression and functional activity of ryanodine receptors in resistant and conduit pulmonary as well as mesenteric artery smooth muscle cells. J Vasc Res 2008; 45: 469-79.

28 Murphy RA. What is special about smooth muscle? The significance of covalent crossbridge regulation. FASEB J 1994; 8: 311-8.

29 Zhou ZL, Ikebe M. New isoforms of $\mathrm{Ca}^{2+} /$ calmodulin-dependent protein kinase II in smooth muscle. Biochem J 1994; 299: 489-95.

30 Raina H, Zacharia J, Li M, Wier WG. Activation by $\mathrm{Ca}^{2+} /$ calmodulin of an exogenous myosin, light chan kinase in mouse arteries. J Physiol 2009; 587: 2599-612.

31 Jackson-Weaver O, Osmond JM, Riddle MA, Naik JS, Gonzalez Bosc LV, Walker BR, et al. Hydrogen sulfide dilates rat mesenteric arteries by activating endothelial large-conductance $\mathrm{Ca}^{2+}$-activated $\mathrm{K}^{+}$channels and smooth muscle $\mathrm{Ca}^{2+}$ sparks. Am J Physiol Heart Circ Physiol 2013; 304: H1446-54.

32 Kai L, Wang ZF, Hu DY, Shi YL, Liu LM. Opioid receptor antagonists modulate $\mathrm{Ca}^{2+}$-activated $\mathrm{K}^{+}$channels in mesenteric arterial smooth muscle cells of rats in hemorrhagic shock. Shock 2003; 19: 85-90. 